\begin{tabular}{|c|c|c|}
\hline 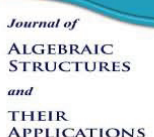 & $\begin{array}{l}\text { Journal of Algebraic Structures and Their Applications } \\
\qquad \text { ISSN: } 2382-9761\end{array}$ & \\
\hline-1 & www.as.yazd.ac.ir & \\
\hline
\end{tabular}

Algebraic Structures and Their Applications Vol. 6 No. 1 ( 2019 ) pp 47-54.

\title{
GRAPH PRODUCT OF GENERALIZED CAYLEY GRAPHS OVER POLYGROUPS
}

\author{
D. HEIDARI* AND B. DAVVAZ
}

\begin{abstract}
In this paper, we introduce a suitable generalization of Cayley graphs that is defined over polygroups (GCP-graph) and give some examples and properties. Then, we mention a generalization of NEPS that contains some known graph operations and apply to GCP-graphs. Finally, we prove that the product of GCP-graphs is again a GCP-graph.
\end{abstract}

\section{INTRODUCTION AND PRELIMINARIES}

A graph product is a binary operation on graphs. So, many large graphs can be constructed from existing smaller graphs. In [IU], Li et al. studied the properties of the lexicographic product of vertex-transitive and of edge-transitive graphs, and of the Cayley graphs. They proved that the lexicographic product of vertex-transitive (edge-transitive) graphs is a vertextransitive (edge-transitive) graph and, in particular, the lexicographic product of Cayley graphs is a Cayley graph.

DOI : $10.29252 /$ as . 2019.1340

MSC(2010): Primary: 20N20, 05C25

Keywords: Simple graph, Caylay graph, polygroup, GCP-graph, graph product.

Received:22 Nov 2018, Accepted: 17 Feb 2019.

*Corresponding author

(C) 2019 Yazd University. 
The non-complete extended p-sum (NEPS) of graphs is very general graph operation. Many graph operations are special cases of NEPS, to name just the sum, product and strong product of graphs.

Definition 1.1. Let $B \subseteq\{0,1\}^{n} \backslash\{(0,0, \cdots, 0)\}$ be a set of binary $n$-tuples. NEPS with basis $B$ of graphs $G_{1}, \ldots, G_{n}$ is the graph whose vertex set is the Cartesian product of the vertex sets of graphs $G_{1}, \ldots, G_{n}$ in which two vertices, say $\left(x_{1}, \cdots, x_{n}\right)$ and $\left(y_{1}, \cdots, y_{n}\right)$, are adjacent if and only if there exists an $n$-tuple $\left(\beta_{1}, \cdots, \beta_{n}\right) \in B$ such that $x_{i}=y_{i}$ holds whenever $\beta_{i}=0$, and $x_{i}$ is adjacent to $y_{i}\left(\right.$ in $G_{i}$ ) whenever $\beta_{i}=1$.

Klotz and Sander proved that the class of gcd-graphs and the class of NEPS of complete graphs coincide [14].

The theory of hypergroups which is a generalization of the concept of ordinary groups first was introduced by Marty [15]. Since then many researchers have worked on hypergroups and developed it. A short review of this theory appears in [ [5, $\mathbf{6}, \mathbf{z}, \mathbf{9}, \mathbf{4}]$ ]. Application of hypergroups has mainly appeared in special subclasses. For example, polygroups which are certain subclasses of hypergroups are studied in [13] by Ioulidis and are used to study color algebra [3, 4]. Quasi-canonical hypergroups (called polygroups by Comer) were introduced in [2], as a generalization of canonical hypergroups, introduced in [I6]. In [II] Heidari et al. studied the concept of topological polygroups as a generalization of topological groups.

Definition 1.2. [3, 9] A polygroup is a system $\langle P, \circ, 1,-1\rangle$, where $1 \in P,-1$ is a unitary operation on $P$, $\circ$ maps $P \times P$ into the non-empty subsets of $P$, and the following axioms hold for all $x, y, z \in P$ :

$(\mathrm{P} 1) x \circ(y \circ z)=(x \circ y) \circ z$,

$(\mathrm{P} 2) 1 \circ x=x=x \circ 1$,

(P3) $x \in y \circ z$ implies $y \in x \circ z^{-1}$ and $z \in y^{-1} \circ x$.

If $A, B$ are non-empty subsets of $P$, then $A \circ B$ is given by

$$
A \circ B=\bigcup_{\substack{a \in A \\ b \in B}} a \circ b .
$$

$x \circ A$ is used for $\{x\} \circ A$ and $A \circ x$ for $A \circ\{x\}$. Clearly, every group is a polygroup. The following elementary facts about polygroups follow easily from the axioms: $1 \in x \circ x^{-1} \cap x^{-1} \circ x$, $1^{-1}=1,\left(x^{-1}\right)^{-1}=x$, and $(x \circ y)^{-1}=y^{-1} \circ x^{-1}$, where $A^{-1}=\left\{a^{-1} \mid a \in A\right\}$. A polygroup in which every element has order 2 (i.e., $x^{-1}=x$ for all $\left.x\right)$ is called symmetric. For more details about polygroups we refer the readers to $[9]$.

Lemma 1.3. Let $\mathbf{P}=\left\langle P, \circ, 1,^{-1}\right\rangle$ be a polygroup and $B$ be a non-empty subset of $P$. Then

$$
\langle B\rangle=\left\{x \in P: x \in b_{1} \circ b_{2} \circ \cdots \circ b_{k} \text {, s.t. } \mathrm{k} \in \mathbb{N}, \mathrm{b}_{\mathrm{i}} \in \mathrm{B} \cup \mathrm{B}^{-1}\right\} \text {. }
$$


Proof. It is straightforward.

\section{MAin RESUlts}

It is easy to see that the Cartesian, tensor and strong product of graphs are special types of NEPS but the lexicographic product is not. In this section, we mention a generalization of NEPS that contain some other known graph operations, for example the compliment and the lexicographic product of graphs. Firstly, we introduce a suitable generalization of Cayley graphs that is defined over polygroups.

Definition 2.1. [ [ $[2]$ Let $\mathbf{P}=\left\langle P, \circ, 1,{ }^{-1}\right\rangle$ be a polygroup and $S$, say the connection set, be a non-empty inverse closed subset (i.e. $S^{-1}=S$ ) of $P$. Then we define the generalized Cayley graph $\operatorname{GCP}(\mathbf{P} ; \mathrm{S})$ as a simple graph with vertex set $P$ and edge set

$$
E=\left\{\{x, y\} \mid x \neq y \text { and } x \circ y^{-1} \cap S \neq \emptyset\right\} .
$$

A graph $\Lambda$ is called a GCP-graph if there exists a polygroup $\mathbf{P}$ and a connection set $S$ such that $\Lambda \cong \operatorname{GCP}(\mathbf{P} ; \mathrm{S})$.

In what follows, we provide some examples of generalized Cayley graphs with the given polygroups and connection sets.

Example 2.2. The generalized Cayley graph of the polygroup $\mathbf{P}_{\mathbf{2}}=\left\langle\{1,2,3,4\}, \circ, 1,{ }^{-1}\right\rangle$ and the connection set $\{3,4\}$ is shown in Figure [2.2.

\begin{tabular}{c|cccc}
$\circ$ & 1 & 2 & 3 & 4 \\
\hline 1 & 1 & 2 & 3 & 4 \\
2 & 2 & 1 & 4 & 3 \\
3 & 3 & 4 & $\{1,3\}$ & $\{2,4\}$ \\
4 & 4 & 3 & $\{2,4\}$ & $\{1,3\}$
\end{tabular}

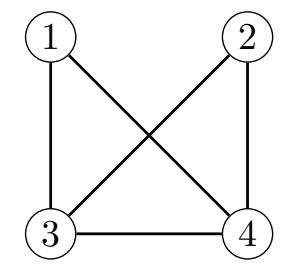

Figure 1. $\operatorname{GCP}\left(\mathbf{P}_{\mathbf{2}} ;\{3,4\}\right)$ 
Example 2.3. The generalized Cayley graph of the polygroup $\mathbf{P}_{\mathbf{3}}=\left\langle\{1,2,3,4,5\}, \circ, 1,{ }^{-1}\right\rangle$ and the connection set $\{3,4\}$ is shown in Figure 2.3 .

\begin{tabular}{c|ccccc}
$\circ$ & 1 & 2 & 3 & 4 & 5 \\
\hline 1 & 1 & 2 & 3 & 4 & 5 \\
2 & 2 & $\{1,2\}$ & 3 & 4 & 5 \\
3 & 3 & 3 & $\{1,2,4\}$ & $\{3,5\}$ & $\{4,5\}$ \\
4 & 4 & 4 & $\{3,5\}$ & $\{1,2,4,5\}$ & $\{3,4\}$ \\
5 & 5 & 5 & $\{4,5\}$ & $\{3,4\}$ & $\{1,2,3,5\}$
\end{tabular}

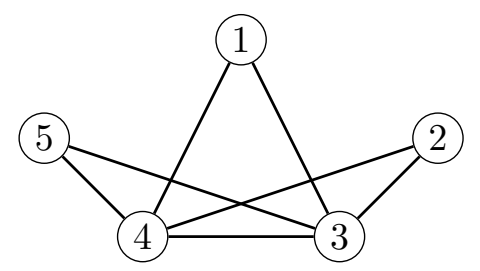

Figure 2. $\operatorname{GCP}\left(\mathbf{P}_{\mathbf{3}} ;\{3,4\}\right)$

The necessary and sufficient condition for connectedness of a GCP-graph over a polygroup is same as that in Cayley graphs. In other words:

Theorem 2.4. [I2] Let $\mathbf{P}=\left\langle P, \circ, 1,{ }^{-1}\right\rangle$ be a polygroup and $S$ be a connection set. Then, the generalized Cayley graph $\mathrm{GCP}(\mathbf{P} ; \mathrm{S})$ is connected if and only if $S$ generates $P$.

Definition 2.5. Let $G_{1}, G_{2}, \ldots, G_{n}$ be graphs and $B \subseteq\{-1,0,1\}^{n}$. Then the product of $G_{1}, G_{2}, \ldots, G_{n}$ respect to the base $B$, denoted by $G=\operatorname{Pr}\left(G_{1}, \ldots, G_{n} ; B\right)$, is a graph with the vertex set $V(G)=V\left(G_{1}\right) \times \cdots \times V\left(G_{n}\right)$ and two distinct vertices $x=\left(x_{1}, \ldots, x_{n}\right)$ and $y=\left(y_{1} \ldots, y_{n}\right)$ are adjacent in $G$, if and only if there exists an $n$-tuple $\beta=\left(\beta_{1}, \cdots, \beta_{n}\right) \in B$ such that $x_{i}=y_{i}$, whenever $\beta_{i}=0, x_{i}$ and $y_{i}$ are adjacent in $G_{i}$, whenever $\beta_{i}=1$ and $x_{i}$ and $y_{i}$ are non-adjacent in $G_{i}$, whenever $\beta_{i}=-1$.

In particular, for a simple graph $G=(V, E)$, we have:

$$
\begin{gathered}
\operatorname{Pr}(G ; \emptyset)=\operatorname{Pr}(G ;\{0\}) \cong \overline{K_{|V|}} \\
\operatorname{Pr}(G ;\{1\})=G \\
\operatorname{Pr}(G ;\{-1\})=\bar{G}
\end{gathered}
$$

Also, some known product operations on graphs can be considered as the product graphs with suitable bases. 
Lemma 2.6. Let $G_{1}, \ldots, G_{n}$ be simple graphs and $*$ be the Cartesian $(\square)$, tensor $(\times)$, strong $(\otimes)$ or lexicographic product (o) of graphs. Then there exists a base $B \subseteq\{-1,0,1\}^{n}$ such that $G_{1} * \cdots * G_{n}=\operatorname{Pr}\left(G_{1}, \ldots, G_{n} ; B\right)$.

Proof. For every $i \in\{1,2, \ldots, n\}$ put

$$
\Delta_{i}=\{0\}^{i-1} \times\{1\} \times\{0\}^{n-i} \text { and } \Sigma_{i}=\{0\}^{i-1} \times\{1\} \times\{-1,0,1\}^{n-i} .
$$

Then we have

$$
\begin{aligned}
G_{1} \square \cdots \square G_{n} & =\operatorname{Pr}\left(G_{1}, \ldots, G_{n} ; B_{1}\right) ; \\
G_{1} \times \cdots \times G_{n} & =\operatorname{Pr}\left(G_{1}, \ldots, G_{n} ; B_{2}\right) ; \\
G_{1} \otimes \cdots \otimes G_{n} & =\operatorname{Pr}\left(G_{1}, \ldots, G_{n} ; B_{3}\right) ; \\
G_{1} \circ \cdots \circ G_{n} & =\operatorname{Pr}\left(G_{1}, \ldots, G_{n} ; B_{4}\right) ;
\end{aligned}
$$

where $B_{1}=\{(1,1, \cdots, 1)\}, B_{2}=\bigcup_{i=1}^{n} \Delta_{i}, B_{3}=\left(\bigcup_{i=1}^{n} \Delta_{i}\right) \cup\{(1,1, \ldots, 1)\}$ and $B_{4}=\bigcup_{i=1}^{n} \Sigma_{i}$.

In the following theorem we prove that the product of GCP-graphs is a GCP-graph.

Theorem 2.7. Let $G_{1}, G_{2}, \ldots, G_{n}$ be GCP-graphs and $B \subseteq\{-1,0,1\}^{n}$. Then $\operatorname{Pr}\left(\mathrm{G}_{1}, \mathrm{G}_{2}, \ldots, \mathrm{G}_{\mathrm{n}} ; \mathrm{B}\right)$ is a GCP-graph.

Proof. Suppose that $G_{1}, G_{2}, \ldots, G_{n}$ are GCP-graphs, $B \subseteq\{-1,0,1\}^{n}$ and $G=$ $\operatorname{Pr}\left(\mathrm{G}_{1}, \mathrm{G}_{2}, \ldots, \mathrm{G}_{\mathrm{n}} ; \mathrm{B}\right)$. Thus, there exist polygroups

$$
\mathbf{P}_{\mathbf{1}}=\left\langle P_{1}, \circ_{1}, e_{1},{ }_{1}\right\rangle, \mathbf{P}_{\mathbf{2}}=\left\langle P_{2}, \circ_{2}, e_{2},{ }_{2}\right\rangle, \ldots, \mathbf{P}_{\mathbf{n}}=\left\langle P_{n}, \circ_{n}, e_{n},{ }^{I_{n}}\right\rangle
$$

and connection sets $S_{1} \subseteq P_{1}, S_{2} \subseteq P_{2}, \ldots, S_{n} \subseteq P_{n}$ such that

$$
G_{i} \cong \Gamma_{i}=\operatorname{GCP}\left(\mathbf{P}_{\mathbf{i}} ; \mathrm{S}_{\mathrm{i}}\right)
$$

for every $i=1,2, \ldots, n$.

Let $\Gamma=\operatorname{Pr}\left(\Gamma_{1}, \Gamma_{2}, \ldots, \Gamma_{n} ; B\right)$ and $\Lambda=\operatorname{GCP}\left(\mathbf{P}_{\mathbf{1}} \times \mathbf{P}_{\mathbf{2}} \times \cdots \times \mathbf{P}_{\mathbf{n}}, S\right)$ such that

$$
S=\bigcup_{\beta \in B}\left(S_{1}^{\left(\beta_{1}\right)} \times S_{2}^{\left(\beta_{2}\right)} \times \cdots \times S_{2}^{\left(\beta_{n}\right)}\right),
$$

where $S_{i}^{(0)}=\left\{e_{i}\right\}, S_{i}^{(1)}=S_{i}$ and $S_{i}^{(-1)}=P_{i} \backslash S_{i}$ for all $i=1,2, \ldots, n$. 
We claim that $\Gamma=\Lambda$. Since, by the definition, $V(\Gamma)=V(\Lambda)=P_{1} \times P_{2} \times \cdots \times P_{n}$ as desired. On the other hand,

$$
\begin{aligned}
E(\Gamma) & =\left\{\left(\left(x_{1}, \ldots, x_{n}\right),\left(y_{1}, \ldots, y_{n}\right)\right) \mid \exists \beta \in B, x_{i}=y_{i} \Leftrightarrow \beta_{i}=0,\right. \\
& \left.x_{i} \sim y_{i} \Leftrightarrow \beta_{i}=1, x_{i} \neq y_{i}, x_{i} \nsim y_{i} \Leftrightarrow \beta_{i}=-1, \forall i \in\{1,2, \ldots, n\}\right\} \\
= & \left\{\left(\left(x_{1}, \ldots, x_{n}\right),\left(y_{1}, \ldots, y_{n}\right)\right) \mid \exists \beta \in B, e_{i} \in x_{i} \circ_{i} y_{i}^{I_{i}} \Leftrightarrow \beta_{i}=0,\right. \\
& \left(x_{i} \circ_{i} y_{i}^{I_{i}}\right) \cap S_{i} \neq \emptyset \Leftrightarrow \beta_{i}=1,\left(x_{i} \circ_{i} y_{i}^{I_{i}}\right) \cap S_{i}=\emptyset \Leftrightarrow \beta_{i}=-1, \\
& \forall i \in\{1,2, \ldots, n\}\} \\
& =\left\{\left(\left(x_{1}, \ldots, x_{n}\right),\left(y_{1}, \ldots, y_{n}\right)\right) \mid \exists \beta \in B,\right. \\
& \left.\left(\left(x_{1} \circ_{1} y_{1}^{I_{1}}\right) \times \cdots \times\left(x_{n} \circ y_{n}^{I_{n}}\right)\right) \cap\left(S_{1}^{\left(\beta_{1}\right)} \times \cdots \times S_{n}^{\left(\beta_{n}\right)}\right) \neq \emptyset\right\} \\
& =E(\Lambda) .
\end{aligned}
$$

Therefore, $G \cong \Gamma=\Lambda$ so the proof is complete since, $\Lambda$ is a GCP-graph.

Corollary 2.8. The Cartesian, tensor, strong and lexicographic product of GCP-graphs are GCP-graphs.

Finally, from Theorem 2.7 we can obtain the connection sets for the Cartesian, tensor, strong and lexicographic product of two GCP-graphs.

Lemma 2.9. Let $\mathbf{P}_{\mathbf{1}}=\left\langle P_{1}, \circ_{1}, e_{1},{ }^{I_{1}}\right\rangle$ and $\mathbf{P}_{\mathbf{2}}=\left\langle P_{2}, \circ_{2}, e_{2},{ }^{I_{2}}\right\rangle$ be two polygroups and $\mathbf{P}=$ $\mathbf{P}_{\mathbf{1}} \times \mathbf{P}_{\mathbf{2}}$. Then for every connection sets $S_{1} \subseteq P_{1}$ and $S_{2} \subseteq P_{2}$ the following assertions hold:

(1) $\operatorname{GCP}\left(\mathbf{P}_{\mathbf{1}}, S_{1}\right) \square \operatorname{GCP}\left(\mathbf{P}_{\mathbf{2}} ; S_{2}\right)=\operatorname{GCP}\left(\mathbf{P} ; S_{1} \times S_{2}\right)$;

(2) $\operatorname{GCP}\left(\mathbf{P}_{\mathbf{1}}, S_{1}\right) \times \operatorname{GCP}\left(\mathbf{P}_{\mathbf{2}} ; S_{2}\right)=\operatorname{GCP}\left(\mathbf{P} ; S_{1} \times\left\{e_{2}\right\} \cup\left\{e_{1}\right\} \times S_{2}\right)$;

(3) $\operatorname{GCP}\left(\mathbf{P}_{\mathbf{1}}, S_{1}\right) \otimes \operatorname{GCP}\left(\mathbf{P}_{\mathbf{2}} ; S_{2}\right)=\operatorname{GCP}\left(\mathbf{P} ; S_{1} \times S_{2} \cup S_{1} \times\left\{e_{2}\right\} \cup\left\{e_{1}\right\} \times S_{2}\right)$;

(4) $\operatorname{GCP}\left(\mathbf{P}_{\mathbf{1}}, S_{1}\right) \circ \operatorname{GCP}\left(\mathbf{P}_{\mathbf{2}} ; S_{2}\right)=\operatorname{GCP}\left(\mathbf{P} ; S_{1} \times P_{2} \cup\left\{e_{1}\right\} \times S_{2}\right)$.

In spite of the fact that every Cayley graph is a GCP-graph and so the following proposition holds as a result of Lemma [2.9, we prove it independently for the lexicographic product. This proof is modified the proof of Theorem 2.4 in [10]].

Proposition 2.10. The Cartesian, tensor, strong and lexicographic product of Cayley graphs are Cayley graphs.

Proof. Let $\Gamma_{1}=\operatorname{Cay}\left(G_{1}, S_{1}\right)$ and $\Gamma_{1}=\operatorname{Cay}\left(G_{1}, S_{1}\right)$ be two Cayley graphs. Consider $\Gamma=$ $\Gamma_{1} \circ \Gamma_{2}$ and $\Lambda=\operatorname{Cay}\left(G_{1} \times G_{2}, S\right)$ where, $S=S_{1} \times G_{2} \cup\left\{e_{1}\right\} \times S_{2}$. Then $\Gamma=\Lambda$ since, $V(\Gamma)=V(\Lambda)=G_{1} \times G_{2}$ and

$$
\begin{aligned}
E(\Gamma) & =\left\{\left(\left(x_{1}, x_{1}\right),\left(y_{1}, y_{2}\right)\right) \mid x_{1} \sim y_{1} \text { or }\left(x_{1}=y_{1} \text { and } x_{2} \sim y_{2}\right)\right\} \\
& =\left\{\left(\left(x_{1}, x_{1}\right),\left(y_{1}, y_{2}\right)\right) \mid x_{1} y_{1}^{-1} \in S_{1} \text { or } x_{1} y_{1}^{-1}=e_{1} \text { and } x_{2} y_{2}^{-1} \in S_{2}\right\} \\
& =\left\{\left(\left(x_{1}, x_{1}\right),\left(y_{1}, y_{2}\right)\right) \mid\left(x_{1}, x_{2}\right)\left(y_{1}, y_{2}\right)^{-1} \in S\right\}=E(\Lambda) .
\end{aligned}
$$


Therefore, the proof is complete.

Example 2.11. Let $K_{2}$ be the complete graph on two vertises and $H$ be the chair graph. Then $K_{2} \cong \operatorname{Cay}\left(\mathbb{Z}_{2},\{1\}\right)$ and $H \cong \operatorname{GCP}\left(\mathbf{P}_{\mathbf{3}},\{5\}\right)$, where $\mathbf{P}_{\mathbf{3}}$ is the polygroup considered in Exapmple 2. The Cartesian product of $K_{2}$ and $H$ is $G=\operatorname{GCP}\left(\mathbb{Z}_{2} \times P_{3},\{(1,5)\}\right)$. See Figure [2.1].
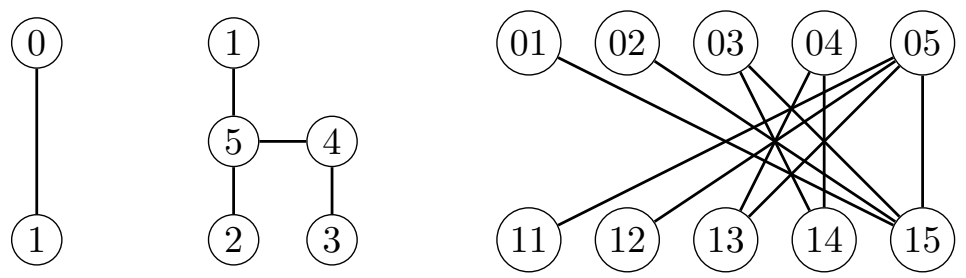

Figure $3 . K_{2} \times H \cong G$

\section{ACKNowledgments}

The authors gratefully thank to referees for the helpful comments and recommendations leading us to improve the readability and quality of the paper.

\section{REFERENCES}

[1] J. A. Bondy, U. S. R. Murthy, Graph Theory with Applications, Macmillan Press Ltd, 1976.

[2] P. Bonansinga and P. Corsini, Sugli omomorfismi di semi-ipergruppi e di ipergruppi, Boll. Un. Mat. Italy, 1-B (1982) 717-727.

[3] S.D. Comer, Polygroups derived from cogroups, J. Algebra, 89 (1984), 397-405.

[4] S.D. Comer, Extension of polygroups by polygroups and their representations using color schemes, Lecture notes in Math., No 1004, Universal Algebra and Lattice Theory, (1982) 91-103.

[5] P. Corsini, Prolegomena of Hypergroup Theory, Aviani Editore, Tricesimo, 1993.

[6] P. Corsini and V. Leoreanu, Applications of Hyperstructure Theory, Kluwer Academical Publications, Dordrecht, 2003.

[7] D. Cvetković, R. Lučić, A new generalization of the p-sum of graphs, Univ. Beograd, Publ. Elektrotehn. Fak., Ser. Mat. Fiz. No. 302 - No. 319 (1970), 67-71.

[8] B. Davvaz, Semihypergroup theory, Elsevier/Academic Press, London, 2016. viii+156 pp.

[9] B. Davvaz, Polygroup Theory and Related Systems, World Scientific Publishing Co. Pte. Ltd., Hackensack, NJ, 2013.

[10] Feng Li, Wei Wang, Zongben Xu and Haixing Zhao, Some results on the lexicographic product of vertextransitive graphs, Appl. Math. Lett. 24(11) (2011), 1924-1926.

[11] D. Heidari, B. Davvaz and S. M. S. Modarres, Topological polygroups, Bull. Malays. Math. Sci. Soc., 39 (2016), 707-721. 
[12] D. Heidari, M. Amooshahi and B. Davvaz, Generalized Cayley graphs over polygroups, Comm. Algebra, (2019), DOI:10.1080/00927872.2018.1530254.

[13] S. Ioulidis, Polygroups et certains de leurs properietes, Bull. Greek Math. Soc., 22 (1981),

[14] W. Klotz, and T. Sander, GCD-graphs and NEPS of complete graphs. Ars Math. Contemp, 6 (2013), no. 2, 289-299.

[15] F. Marty, Sur une generalization de la notion de groupe, $8^{\text {iem }}$ Congress Math. Scandinaves, Stockholm, (1934) 45-49.

[16] J. Mittas, Hypergroupes canoniques, Math. Balkanica, Beograd, 2 (1972) 165-179.

[17] T. Vougiouklis, Hyperstructures and Their Representations, Hadronic Press, Inc, 115, Palm Harber, USA (1994).

\section{Heidari}

Faculty of Science

Mahallat Institute of Higher Education, Mahallat

Markazi, Iran

dheidari82@gmail.com

B. Davvaz

Department of Mathematics

Yazd University, Yazd

Yazd, Iran

davvaz@yazd.ac.ir 